\title{
Avaliação de exames laboratoriais e sintomatologia de pacientes com diagnóstico molecular positivo (RT-qPCR) para COVID-19 internados em Unidade de Terapia Intensiva em um município do Oeste do Paraná
}

\author{
Evaluation of laboratory exams and symptoms of patients with molecular diagnosis ( $R T-q P C R)$ of
} COVID-19 admitted to an Intensive Care Unit in a Western Parana city

Evaluación de pruebas laboratoriales y síntomas de pacientes con diagnóstico molecular (RTqPCR) para COVID-19 ingresados en una Unidad de Cuidados Intensivos en una ciudad del oeste de Paraná

\section{Resumo}

A pandemia desencadeada pela COVID-19 vem assolando o mundo desde dezembro de 2019 pois sem nenhum tratamento específico conhecido houve o óbito quase 3 milhões de indivíduos no mundo. Sua transmissão ocorre de pessoa para pessoa e seus principais sintomas são febre, tosse seca e fadiga. O objetivo desse estudo foi analisar o prognóstico de pacientes com SARS-CoV-2, internados em uma UTI do Oeste do PR, através dos parâmetros hematológicos e sintomatológicos e assim auxiliar no estabelecimento de protocolos e condutas. Dos dados obtidos, verificou-se maior número de óbitos entre pacientes idosos $(\mathrm{p}=0,01)$ e o perfil laboratorial de pior prognóstico foi aquele com hematocritopenia, leucocitose, linfocitopenia e neutrocitose, além da maior associação à sintomas respiratórios. Também se constatou a não associação do sexo com as chances de óbito $(p=0,13)$ ou tempo de internação $(p=0,36)$. Sendo assim, novos estudos podem utilizar desses marcadores laboratoriais e sintomatológicos, assim como da idade, para auxiliar na rotina médica frente a pandemia.

Palavras-chave: COVID-19; SARS-CoV-2; Prognóstico; Marcadores laboratoriais; Sintomatologia; Mortalidade.

\section{Abstract}

The pandemic triggered by COVID-19 has been plaguing the world since December 2019, without any known specific treatment, leading to nearly 3 million deaths worldwide. Its transmission occurs from person to person and its main 
symptoms are fever, dry cough and fatigue. The aim of this study was to analyze the prognosis of patients with SARSCoV-2 admitted to an Intensive Care Unit in the West region of Parana, through hematological and symptomatological parameters, helping in the establishment of protocols and procedures. From the data obtained, there was a greater number of deaths among older patients $(\mathrm{p}=0,01)$, and the laboratory profile with the worst prognosis was with hematocritopenia, leukocytosis, lymphocytopenia, neutrocytosis and greater number of respiratory symptoms. There was no association between sex and death $(\mathrm{p}=0,13)$ or length of stay $(\mathrm{p}=0,36)$. Therefore, new studies can use these laboratory and symptomatological markers, as well as age, to assist in the pandemic medical routine.

Keywords: COVID-19; SARS-CoV-2; Prognosis; Laboratory markers; Symptomatology; Mortality.

\section{Resumen}

La pandemia desencadenada por COVID-19 ha estado asolando al mundo desde diciembre de 2019. Sin ningún tratamiento específico conocido, provocó la muerte de casi 3 millones de personas en todo el mundo. Su transmisión ocurre de persona a persona y sus principales síntomas son fiebre, tos seca y fatiga. El objetivo de este estudio fue analizar el pronóstico de pacientes con SARS-CoV-2, ingresados en una Unidad de Terapia Intensiva del oeste de Paraná, a través de parámetros hematológicos y sintomatológicos, y así ayudar en el establecimiento de protocolos y procedimientos. A partir de los datos obtenidos, hubo un mayor número de muertes entre los pacientes ancianos $(\mathrm{p}=0,01)$, y el perfil de laboratorio con peor pronóstico fue el de hematocritopenia, leucocitosis, linfocitopenia y neutrocitosis, además de una mayor asociación con síntomas respiratorios. Tampoco hubo asociación entre el sexo y las posibilidades de muerte $(\mathrm{p}=0,13)$ o la duración de la estancia hospitalaria $(\mathrm{p}=0,36)$. Por tanto, nuevos estudios pueden utilizar estos marcadores de laboratorio y sintomatológicos, asi como la edad, para ayudar en la rutina médica ante la pandemia.

Palabras clave: COVID-19; SARS-CoV-2; Pronóstico; Marcadores de laboratorio; Sintomatología; Mortalidad.

\section{Introdução}

Em dezembro de 2019, a Síndrome Respiratória Aguda e Severa por Coronavirus 2 (SARS-CoV-2), causadora da doença conhecida como Coronavirus 2019 (COVID-19), surgiu como um surto epidêmico em Wuhan na China (Zhu et al., 2020) e progrediu rapidamente, sendo oficialmente considera pela Organização Mundial de Saúde (OMS), em março de 2020, uma pandemia mundial (OPAS, 2020).

Em todo o mundo, até 22 de janeiro de 2021, foram registrados 96.267.473 casos confirmados de COVID-19 e 2.082.745 mortes (OPAS, 2020). A rápida disseminação da doença tem causado danos relevantes à saúde pública e a economia (Lai et al., 2020; Wang et al., 2020). Bilhões de pessoas sofreram com os impactos das medidas de distanciamento social e com as consequências socioeconômicas da pandemia (Terpos et al., 2020).

A transmissão da COVID-19 ocorre de pessoa para pessoa, através de contato com gotículas de saliva, espirro, tosse e catarro, acompanhado de contato com a boca, nariz e olhos (OPAS, 2020). As manifestações clínicas mais comuns são febre, tosse seca e fadiga (Xie et al., 2020). Contudo, seu padrão de transmissibilidade, infectividade e mortalidade, bem como o tratamento específico, ainda não estão bem definidos (Pereira, et al., 2020; Xie et al., 2020).

A entrada do SARS-CoV-2 nas células humanas hospedeiras, é mediada pelo receptor celular da enzima conversora de angiotensina 2 (ECA 2) (Li, Bai, \& Hashikawa, 2020; Terpos et al., 2020), presente na mucosa oral e nasal, pulmões, rins, intestino e cérebro (Hamminig et al., 2004). O mecanismo fisiopatológico do vírus envolve a dissociação do ferro do grupo heme da hemoglobina, formando porfirina, que reduziria o transporte de oxigênio e dióxido de carbono pela hemoglobina levando a sintomas respiratórios ( $\mathrm{Liu} \& \mathrm{Li}, 2020$ ).

A COVID-19 é considerada uma doença sistêmica, a literatura da área tem demostrado significativas repercussões no sistema hematopoiético e na hemóstase (Driggin et al., 2020; Liu \& LI, 2020; Terpos et al., 2020). Indivíduos infectados pela COVID-19 frequentemente apresentam aumento e queda tanto do tempo de protrombina como do tempo de tromboplastina parcial ativada (sugerindo ativação da coagulação e coagulopatia consumptiva) (Harenberg \& Favaloro, 2020). A hipercoagulação sanguínea também é um achado comum em pacientes com COVID-19 hospitalizados (Terpos et al., 2020). A linfopenia, a razão neutrófilos/linfócitos e a relação pico de plaqueta/linfócito podem ter um valor de prognóstico, principalmente em casos graves (Terpos et al., 2020). 
Idosos e pessoas com comorbidades possuem maior risco de morte por COVID-19, contudo é possível que mesmo jovens sem doenças de base, desenvolvam complicações eventualmente letais, como miocardite fulminante e coagulação intravascular disseminada (CIVD) (Madjid et al., 2020; Tang et al., 2020).

Assim, no decurso da doença, avaliações hematológicas longitudinais têm potencial de auxílio no prognóstico e no processo decisório para intervenções imediatas (Terpos et al., 2020). Neste sentido, o presente estudo tem como objetivo analisar as relações prognósticas e possíveis inferências diagnósticas (como na Dengue) por meio dos parâmetros apresentados no hemograma e da sintomatologia clínica nos primeiros quatorze dias de internamento dos pacientes com diagnóstico de COVID19.

Tendo em vista a magnitude epidemiológica da COVID-19 e o incipiente número de estudos nacionais sobre os parâmetros hematológicos de pacientes acometidos por esta patologia, espera-se contribuir com dados empiricamente relevantes para o estabelecimento de relações entre o hemograma e o desfecho clínico, a fim de auxiliar profissionais de saúde no estabelecimento de protocolos e condutas resolutivas.

\section{Metodologia}

Trata-se de um estudo observacional, analítico e transversal com abordagem quantitativa (Estrela, 2018). Este projeto foi aprovado pelo Comitê de Ética em Pesquisa do Setor de Saúde da Universidade Federal do Paraná sob o parecer consubstanciado CAAE 37305820.1.0000.0102. Todas as considerações éticas requisitadas foram cumpridas pelos pesquisadores. No período de novembro/2020 a janeiro/2021 foram coletados dados laboratoriais e sintomatológicos de 55 indivíduos com idade entre 20 e 90 anos, de ambos os sexos, com diagnóstico molecular (RT-qPCR) positivo para COVID-19, internados na UTI de um hospital público localizado no oeste do Paraná, Brasil. As informações foram coletadas diariamente durante todo o período de internamento dos pacientes. As variáveis coletadas encontram-se disponíveis nas Tabelas 1 e 2.

Tabela 1: Sinais e sintomas COVID-19 internados em um hospital no oeste paranaense em 2020.

\section{Sinais/Sintomas*}

Febre (acima de $38,2^{\circ} \mathrm{C}$ )

Tosse seca

Tosse produtiva

Mialgia

Adinamia

Dor de garganta

Diarreia

Dor abdominal

Precordialgia
Dispneia referida

Dispneia importante/uso de oxigênio

Dispneia importante/Intubação Orotraqueal

Síndrome do olho vermelho

Hisposmia/Anosmia

Cefaleia

Déficit Neurológico

Erupção cutânea

* Coletados diariamente durante o período de internação. Fonte: Autores (2021). 
Tabela 2: Informações laboratoriais coletadas de indivíduos com diagnóstico molecular positivo (RT-qPCR) para COVID19.

\section{Dados laboratoriais*}
Hemácias
Leucócitos
Linfócitos (total e relativo)
Neutrófilos (total e relativo)
Plaquetas

* Coletados diariamente durante o período de internação. Fonte: Autores (2021).

Para avaliação de associação estatística entre as variáveis foi utilizado o software RStudio na versão 1.4.1103. Foram utilizados os testes Qui-Quadrado e de Fisher para avaliação da diferença entre razão de chances e prevalência entre grupos e testes T-Student e Mann-Whitney na comparação da diferença de médias entre grupos. Foi considerado nível de significância $\alpha=0,05$ para rejeitar a hipótese nula e considerar correta a hipótese alternativa.

\section{Resultados e Discussão}

A maioria dos pacientes internados na UTI do hospital deste estudo foi do sexo masculino (56\%). Quarenta e quatro pacientes $(80 \%)$ receberam alta hospitalar, sendo a maior mortalidade em indivíduos do sexo feminino (29\%). Cerca de $40 \%$ dos indivíduos hospitalizados neste estudo apresentaram idade superior a 60 anos, que corresponde à faixa etária com maior risco de mortalidade na infecção pelo SARS-CoV-2 (Tabela 3).

Os sintomas com maior incidência foram adinamia (98\%), dispneia referida (95\%) e tosse produtiva (60\%). Dezessete indivíduos necessitaram de uso de oxigênio suplementar (31\%) e onze (20\%) necessitaram de intubação orotraqueal (IOT) em pelo menos um dia durante o período de internação hospitalar (Tabela 3).

Na Tabela 4 são citados os sintomas dos indivíduos internados por COVID-19 que apresentaram associações com significância estatística $(\alpha<0,05)$. Os indivíduos com idade igual ou superior a 60 anos apresentaram mais hematocritopenia $(\mathrm{p}=0,03)$, linfocitopenia $(\mathrm{p}<0,001)$, neutrocitose $(\mathrm{p}<0,001)$, sinais/sintomas respiratórios $(\mathrm{p}<0,001)$ e sinais/sintomas do Sistema Nervoso Central (SNC) $(\mathrm{p}<0,001)$ na comparação com pacientes com idade inferior a 60 anos. Pacientes que tiveram evolução para óbito apresentaram mais hematocritopenia $(p=0,03)$, leucocitose $(p<0,001)$, linfocitopenia $(p=0,01)$, neutrocitose $(p<0,001)$ e sinais/sintomas respiratórios ( $\mathrm{p}<0,001)$. Por fim, pacientes do sexo masculino tiveram mais hematocritocitose ( $<<0,001)$, linfocitopenia $(p<0,01)$, neutrocitose $(p=0,02)$, plaquetose $(p=0,01)$, menos sinais/sintomas respiratórios $(p=0,006)$ e mais sinais/sintomas gastrointestinais $(\mathrm{p}<0,001)$.

Não houve associação entre sexo e desfecho óbito $(\mathrm{p}=0,13)$, sexo e quantidade de dias em internamento hospitalar ( $\mathrm{p}=0,36)$, contudo os pacientes que evoluíram para óbito apresentaram média de idade estatisticamente superior a aqueles que obtiveram alta hospitalar $(\mathrm{p}=0,01)$. 
Tabela 3: Informações descritivas de indivíduos com diagnóstico molecular positivo (RT-qPCR) para COVID-19 internados em um hospital do oeste paranaense no ano de $2020(n=55)$.

\begin{tabular}{|c|c|c|}
\hline Sexo & Total & $\%$ \\
\hline Feminino & 24 & $43,63 \%$ \\
\hline Masculino & 31 & $56,36 \%$ \\
\hline \multicolumn{3}{|l|}{ Idade } \\
\hline Entre 20 e 39 anos & 8 & $14,54 \%$ \\
\hline Entre 40 e 59 anos & 25 & $45,45 \%$ \\
\hline Entre 60 e 69 anos & 11 & $20,00 \%$ \\
\hline Entre 69 e 79 anos & 5 & $09,09 \%$ \\
\hline Acima de 80 anos & 6 & $10,90 \%$ \\
\hline \multicolumn{3}{|l|}{ Desfecho } \\
\hline Óbito total & 11 & $20,00 \%$ \\
\hline \multicolumn{3}{|l|}{ Óbito por sexo } \\
\hline Feminino & 7 & $63,64 \%$ \\
\hline Masculino & 4 & $36,36 \%$ \\
\hline Alta Hospitalar & 44 & $80,00 \%$ \\
\hline \multicolumn{3}{|l|}{ Incidência de sinais e sintomas } \\
\hline Febre & 16 & $29,09 \%$ \\
\hline Adinamia & 51 & $97,72 \%$ \\
\hline Mialgia & 14 & $25,45 \%$ \\
\hline Erupção Cutânea & 0 & $00,00 \%$ \\
\hline Precordialgia & 5 & $09,09 \%$ \\
\hline Tosse Seca & 33 & $60,00 \%$ \\
\hline Tosse Produtiva & 5 & $09,09 \%$ \\
\hline Dor de garganta & 11 & $20,00 \%$ \\
\hline Dispneia referida & 52 & $94,54 \%$ \\
\hline Dispneia Importante/uso de oxigênio & 17 & $30,90 \%$ \\
\hline Dispneia importante/IOT & 11 & $20,00 \%$ \\
\hline Diarreia & 8 & $14,54 \%$ \\
\hline Dor Abdominal & 12 & $21,81 \%$ \\
\hline Síndrome do olho Vermelho & 1 & $01,81 \%$ \\
\hline Hiposmia/Anosmia & 7 & $12,72 \%$ \\
\hline Cefaleia & 9 & $16,36 \%$ \\
\hline Déficit Neurológico & 4 & $07,27 \%$ \\
\hline
\end{tabular}

Fonte: Autores (2021). 
Tabela 4: Associações estatisticamente relevantes $(\alpha<0,05)$ envolvendo a sintomatologia de indivíduos com COVID-19 em um hospital no Oeste Paranaense no ano de 2020.

\section{Pacientes com idade igual a superior a 60 anos*}

Apresentam mais hematocritopenia $(<36 \%)$

Apresentam mais linfocitopenia $(<1.000)$

Apresentam mais neutrocitose (>70.00)

Apresentam mais sinais/sintomas respiratórios ${ }^{\dagger}$

Apresentam mais sinais/sintomas do $\mathrm{SNC}^{\ddagger}$

\section{Pacientes com desfecho óbito*}

Apresentam mais hematocritopenia $(<36 \%)$

Apresentam mais leucocitose (>10.000)

Apresentam mais linfocitopenia $(<1.000)$

Apresentam mais neutrocitose (>7.000)

Apresentam mais sinais/sintomas respiratórios ${ }^{\dagger}$

\section{Pacientes do sexo masculino*}

Apresentam mais hematocritocitose ( $>46 \%)$

Apresentam mais linfocitopenia $(<1.000)$

Apresentam mais neutrocitose (>7.000)

Apresentam mais plaquetose ( $>400.000)$

Apresentam menos sinais/sintomas respiratórios ${ }^{\dagger}$

Apresentam mais sinais/sintomas gastrointestinais $§$

\section{Odds Ratio}

(IC 95\%)

$\begin{array}{cc}2,21(1,05-4,66) & 0,03 \\ 2,85(1,79-4,53) & <0,001 \\ 2,46(1.53-3,95) & <0,001 \\ 1,55(1,26-1,90) & <0,001 \\ 2,33(1,44-3,79) & <0,001\end{array}$

$2,39(1,10-5,18)$

0,03

$9,33(5,09-17,09)$

$<0,001$

$2,43(1,39-4,24)$

0,01

$9,15(4,91-17,04)$

$<0,001$

$1,82(1,43-2,32)$

$<0,001$

$\begin{array}{lc}3,82(1,85-7,90) & <0,001 \\ 1,88(1,19-2,99) & <0,01 \\ 1,74(1,07-2,84) & 0,02 \\ 3,01(1,20-7,57) & 0,01 \\ 0,76(0,62-0,94) & 0,006 \\ 4,27(1,88-9,71) & <0,001\end{array}$

* Considerando todos os dias de internamento; ${ }^{\dagger}$ Foram considerados os seguintes sinais/sintomas: Tosse seca, tosse produtiva, dor de garganta, dispneia referida e dispneia importante (requerindo intubação orotraqueal e/ou oxigênio); ${ }^{\star}$ Foram considerados os seguintes sinais/sintomas: Síndrome do olho vermelho, hiposmia/anosmia, cefaleia e déficit neurológico; §Foram considerados os seguintes sinais/sintomas: Dor abdominal e diarreia.

Fonte: Autores (2021).

A rápida disseminação da COVID-19, doença causada pelo SARS-CoV-2, resultou em uma crise de saúde pública sem precedentes em todo o mundo, incluindo o Brasil. Este trabalho analisou 55 indivíduos, com diagnóstico molecular (RT-qPCR) positivo para COVID-19, internados em uma UTI de um hospital no oeste do Paraná, Brasil, buscando descrever associações entre idade, sexo e desfecho óbito desses indivíduos com dados laboratoriais e referentes à sintomatologia. As informações laboratoriais dos pacientes, como hematocritopenia, leucocitose, linfocitopenia e neutrocitose foram associadas a pacientes que tiveram desfecho óbito. Dados da literatura apontam que o SARS-CoV-2 desregula a produção de células pela medula óssea. A alteração no número de neutrófilos vêm sendo observado em casos graves de COVID-19, de fato, uma elevação da contagem absoluta de neutrófilos e da razão neutrófilo/linfócito foram relatados nos casos com prognóstico desfavorável, incluindo o óbito (Cheng et al., 2020; Wang et al., 2020).

Qin et al. (2020) descreveram que em pacientes cuja doença avança para o quadro grave, o número de linfócitos T permanece baixo em comparação a pacientes com sintomatologia leve, com a contagem das subpopulações Th1, Th2 e Th17 em 
níveis abaixo do normal. Vários estudos têm relatado que a diminuição de linfócitos T circulantes, incluindo os linfócitos CD4+ e CD8+, está intimamente relacionada à gravidade da COVID-19 (Tan et al., 2020; Wang et al., 2020).

Portanto, o perfil laboratorial do paciente que apresenta como desfecho óbito é aquele com hematocritopenia, leucocitose, linfocitopenia e neutrocitose. Além disso, ocorre associação destes enfermos com maior quantidade de queixas respiratórias. Esses dados estão em consonância com trabalhos na literatura que descreveram que pacientes com quadros severos de COVID-19 apresentam menores parâmetros de hematócrito (hematocritopenia) e linfócitos (linfocitopenia) e maior de neutrófilos (neutrocitose) e leucócitos (leucocitose) na comparação com grupo com sintomas moderados (Wang et al., 2020; Maguire et al, 2020; Wu et al, 2020).

No presente estudo não houve diferença estatisticamente relevante ( $p>0,05)$ referente a contagem de plaquetas no grupo que apresentou desfecho óbito na comparação com desfecho alta hospitalar. Contudo, trabalhos na literatura são divergentes sobre este parâmetro hematológico, pois enquanto Wang et al (2020) não encontrou diferença estatisticamente relevante entre os grupos que apresentaram casos graves daqueles que apresentaram casos moderados, no artigo de Wu et al (2020) casos graves foram associados com menor contagem plaquetária $(\mathrm{p}=0,0043)$.

\section{Conclusão}

Neste estudo houve associação entre maior mortalidade em pacientes com maior idade $(\mathrm{p}=0,01)$, contudo pelo limitado número de participantes não foi possível estratificar, entre os pacientes idosos, aqueles que apresentavam dados laboratoriais dentro dos intervalos de normalidade com aqueles que tinham alteração no hemograma para verificar se também há maior mortalidade, considerando apenas pacientes com idade superior a sessenta anos, com alterações laboratoriais.

Foi cumprido o objetivo do estudo de descrever os marcadores hematológicos e sintomatológicos de pacientes com COVID-19 que tiveram óbito como desfecho. Dessa forma, a relevância deste trabalho é descrever que houve associação estatisticamente relevante de pacientes com hematocritopenia, leucocitose, linfocitopenia, neutrocitose e com maior quantidade de sintomas respiratórios com desfecho óbito.

Estudos futuros podem utilizar marcadores laboratoriais, considerando os primeiros dias de internamento, associados com fatores como sexo e idade para construção de scores que possam predizer a mortalidade de pacientes com COVID-19. Dessa forma, enfermos com maior probabilidade de óbito podem receber cuidados como internação em unidades de terapia intensiva e apoio respiratório com antecedência.

\section{Referências}

Chen, G., Wu, D., Guo, W., Cao, Y., Huang, D., Wang, H., Wang, T., Zhang, X., Chen, H., Yu, H., Zhang, X., Zhang, M., Wu, S., Song, J., Chen, T., Han, M., Li, S., Luo, X., Zhao, J., \& Ning, Q. (2020). Clinical and immunological features of severe and moderate coronavirus disease 2019. Journal of Clinical Investigation, 130(5), 2620-2629. https://doi.org/10.1172/JCI137244

Driggin, E., Madhavan, M. V., Bikdeli, B., Chuich, T., Laracy, J., Biondi-Zoccai, G., Brown, T. S., Der Nigoghossian, C., Zidar, D. A., Haythe, J., Brodie, D., Beckman, J. A., Kirtane, A. J., Stone, G. W., Krumholz, H. M., \& Parikh, S. A. (2020). Cardiovascular Considerations for Patients, Health Care Workers, and Health Systems During the COVID-19 Pandemic. Journal of the American College of Cardiology, 75(18), 2352-2371. https://doi.org/10.1016/j.jacc.2020.03.031

Estrela, C. (2018). Metodologia Científica: ciência, ensino e pesquisa (3ª ed.). Porto Alegre: Artes Médicas.

Hamming, I., Timens, W., Bulthuis, M., Lely, A., Navis, G., \& van Goor, H. (2004). Tissue distribution of ACE2 protein, the functional receptor for SARS coronavirus. A first step in understanding SARS pathogenesis. The Journal of Pathology, 203(2), 631-637. https://doi.org/10.1002/path.1570

Harenberg, J., \& Favaloro, E. (2020). COVID-19: progression of disease and intravascular coagulation - present status and future perspectives. Clinical Chemistry and Laboratory Medicine (CCLM), 58(7), 1029-1036. https://doi.org/10.1515/cclm-2020-0502

Lai, C.-C., Shih, T.-P., Ko, W.-C., Tang, H.-J., \& Hsueh, P.-R. (2020). Severe acute respiratory syndrome coronavirus 2 (SARS-CoV-2) and coronavirus disease2019 (COVID-19): The epidemic and the challenges. International Journal of Antimicrobial Agents, 55(3), 105924. https://doi.org/10.1016/j.ijantimicag.2020.105924

Li, Y., Bai, W., \& Hashikawa, T. (2020). The neuroinvasive potential of SARS-CoV2 may play a role in the respiratory failure of COVID-19 patients. Journal of Medical Virology, 92(6), 552-555. https://doi.org/10.1002/jmv.25728 
Liu W, \& Li, H.. (2020). COVID-19: Attacks the 1-Beta Chain of Hemoglobin and Captures the Porphyrin to Inhibit Human Heme Metabolism. ChemRxiv, 105. https://doi.org/10.26434/chemrxiv.11938173

Madjid, M., Safavi-Naeini, P., Solomon, S. D., \& Vardeny, O. (2020). Potential Effects of Coronaviruses on the Cardiovascular System. JAMA Cardiology, 5(7), 831. https://doi.org/10.1001/jamacardio.2020.1286

Maguire, D., Woods, M., Richards, C., Dolan, R., Veitch, J. W., Sim, W. M. J., Kemmett, O. E. H., Milton, D. C., Randall, S. L. W., Bui, L. D., Goldmann, N., Cameron, A., Laird, B., Talwar, D., Godber, I., Davidson, A., \& McMillan, D. C. (2020). Prognostic factors in patients admitted to an urban teaching hospital with COVID-19 infection. Journal of Translational Medicine, 18(1), 354. https://doi.org/10.1186/s12967-020-02524-4

Organização Pan-americana da Saúde (OPAS). (2020). Folha informativa - COVID-19 (doença causada pelo novo coronavírus). https://www.paho.org/bra/index.php?option=com_content $\&$ view=article\&id=6101: covid19\&Itemid=875

Pereira, M. D., Oliveira, L. C. de, Costa, C. F. T., Bezerra, C. M. de O., Pereira, M. D., Santos, C. K. A. dos, \& Dantas, E. H. M. (2020). A pandemia de COVID19, o isolamento social, consequências na saúde mental e estratégias de enfrentamento: uma revisão integrativa. Research, Society and Development, 9(7), e652974548. https://doi.org/10.33448/rsd-v9i7.4548

Qin, C., Zhou, L., Hu, Z., Zhang, S., Yang, S., Tao, Y., Xie, C., Ma, K., Shang, K., Wang, W., \& Tian, D.-S. (2020). Dysregulation of Immune Response in Patients With Coronavirus 2019 (COVID-19) in Wuhan, China. Clinical Infectious Diseases, 71(15), 762-768. https://doi.org/10.1093/cid/ciaa248

Tan, L., Wang, Q., Zhang, D., Ding, J., Huang, Q., Tang, Y.-Q., Wang, Q., \& Miao, H. (2020). Lymphopenia predicts disease severity of COVID-19: a descriptive and predictive study. Signal Transduction and Targeted Therapy, 5(1), 33. https://doi.org/10.1038/s41392-020-0148-4

Tang, N., Li, D., Wang, X., \& Sun, Z. (2020). Abnormal coagulation parameters are associated with poor prognosis in patients with novel coronavirus pneumonia. Journal of Thrombosis and Haemostasis, 18(4), 844-847. https://doi.org/10.1111/jth.14768

Terpos, E., Ntanasis-Stathopoulos, I., Elalamy, I., Kastritis, E., Sergentanis, T. N., Politou, M., Psaltopoulou, T., Gerotziafas, G., \& Dimopoulos, M. A. (2020). Hematological findings and complications of COVID-19. American Journal of Hematology, 95(7), 834-847. https://doi.org/10.1002/ajh.25829

Wang, C., Deng, R., Gou, L., Fu, Z., Zhang, X., Shao, F., Wang, G., Fu, W., Xiao, J., Ding, X., Li, T., Xiao, X., \& Li, C. (2020). Preliminary study to identify severe from moderate cases of COVID-19 using combined hematology parameters. Annals of Translational Medicine, 8(9), 593-593. https://doi.org/10.21037/atm-20-3391

Wang, D., Hu, B., Hu, C., Zhu, F., Liu, X., Zhang, J., Wang, B., Xiang, H., Cheng, Z., Xiong, Y., Zhao, Y., Li, Y., Wang, X., \& Peng, Z. (2020). Clinical Characteristics of 138 Hospitalized Patients With 2019 Novel Coronavirus-Infected Pneumonia in Wuhan, China. JAMA, 323(11), 1061. https://doi.org/10.1001/jama.2020.1585

Wang, J., Jiang, M., Chen, X., \& Montaner, L. J. (2020). Cytokine storm and leukocyte changes in mild versus severe SARS-CoV-2 infection: Review of 3939 COVID-19 patients in China and emerging pathogenesis and therapy concepts. Journal of Leukocyte Biology, 108(1), 17-41. https://doi.org/10.1002/JLB.3COVR0520-272R

Wu, Y., Huang, X., Sun, J., Xie, T., Lei, Y., Muhammad, J., Li, X., Zeng, X., Zhou, F., Qin, H., Shao, L., \& Zhang, Q. (2020). Clinical Characteristics and Immune Injury Mechanisms in 71 Patients with COVID-19. MSphere, 5(4). https://doi.org/10.1128/mSphere.00362-20

Xie, J., Ding, C., Li, J., Wang, Y., Guo, H., Lu, Z., Wang, J., Zheng, C., Jin, T., Gao, Y., \& He, H. (2020). Characteristics of patients with coronavirus disease (COVID-19) confirmed using an IgM-IgG antibody test. Journal of Medical Virology, 92(10), 2004-2010. https://doi.org/10.1002/jmv.25930

Zhu, N., Zhang, D., Wang, W., Li, X., Yang, B., Song, J., Zhao, X., Huang, B., Shi, W., Lu, R., Niu, P., Zhan, F., Ma, X., Wang, D., Xu, W., Wu, G., Gao, G. F., \& Tan, W. (2020). A Novel Coronavirus from Patients with Pneumonia in China, 2019. New England Journal of Medicine, 382(8), 727-733. https://doi.org/10.1056/NEJMoa2001017 